\title{
DAMAGE TO SEEDS BY THE WORKING BODIES OF CONTINUOUS MACHINES
}

\section{MAIA VIKTOROVNA SUKHANOVA \& VIKTOR PETROVICH ZABRODIN}

Department of Mechanical Engineering, University of Mosul, Iraq

This article deals with the analysis of damaging effect of pre-sowing technological processes on seeds treated with continuous machines, subject to macro and micro damages incurred by the working bodies. Various process flow diagrams with the participation of machinery with various working bodies are considered. The analysis of the pre-sowing technological process with continuous machines showed that screw working bodies have the greatest impact on seed damage during pre-sowing treatment. Damage to the seeds by screw working bodies ranges from 34.6 to $50.0 \%$ of the treated seeds. To reduce the loss of seeds of grain and leguminous crops it is necessary to exclude the use of screw working bodies in pre-sowing treatment of seeds.

KEYWORDS: Pre-sowing Seed Treatment, Seed Damage, Screw Working Body, Cereals and Legumes \& Technological Process
\end{abstract}

Received: Aug 15, 2019; Accepted: Aug 30, 2019; Published: Sep 14, 2019; Paper Id.: IJMPERDOCT201932

\section{INTRODUCTION}

Technological processes of seed dressing machines are diverse and are determined by the design of transporting, metering, and mixing working bodies, the method of dressing (dry or wet), productivity, and cyclical process (continuous or batch).

\section{METHODS}

The analysis of the technological processes of pre-sowing treatment of seeds with continuous machines will be performed subjected to the macro- and micro-damages of seeds. The technological process will be considered as optimal if the total output loss of particles as a result of damage is minimal, that is,

$$
\Sigma \Delta \Pi \rightarrow \min
$$

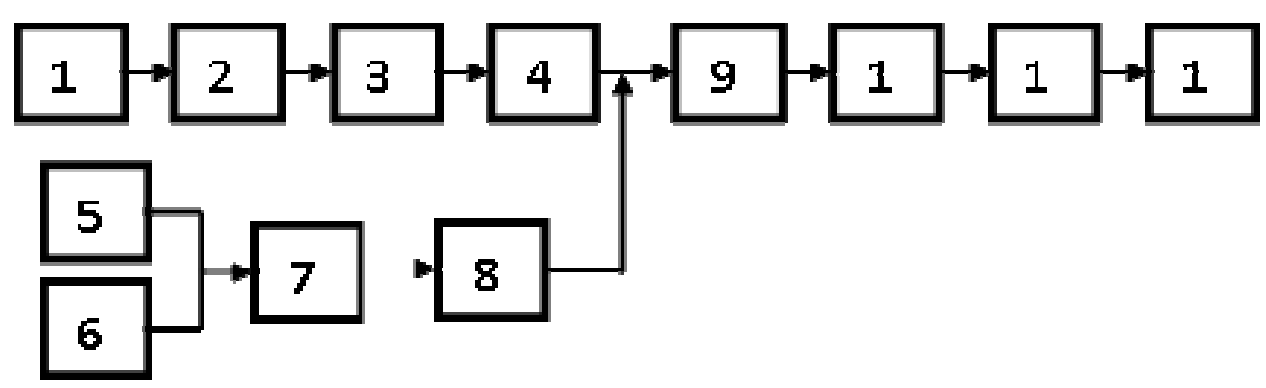

Figure 1: General Block Diagram of the Technological Processes of Seed Dressing. 
In general, the technological process of seed dressing machines includes the following operations (Figure 1).

- $\quad$ withdrawal of seeds from the clamp;

- transportation of material to the storage hopper;

- dosing of seeds;

- feeding of seeds into the mixing chamber;

- $\quad$ dosing of the dressing agent;

- $\quad$ dosing of liquid;

- $\quad$ preparation of a dressing product;

- feeding the dressing product into the mixing chamber;

- $\quad$ mixing of components;

- transportation of dressed seeds;

- removal of excess fluid;

- loading of the treated seeds into the vehicle.

The processes (5-6-7-8) of preparing and feeding the dressing product into the mixing chamber are carried out in parallel with the processes (1-2-3-4) of collecting seeds from the clamp, transporting to the storage hopper, and their dosing.

Each operation of the technological process can be carried out by several types of working bodies. In this case, the working bodies of the machines, performing the same functions and similar in design, can be made of materials with different physical and mechanical properties.

To substantiate the optimal scheme of the seed treatment process, it is necessary to evaluate the influence of the working bodies on the damage to the seeds.

\section{THE MAINPART}

To analyze the influence of the working bodies of the machines on the damage to seeds, we shall consider the most general case of the technological process of seed dressing and present it in the form of a directed diagram (Figure 2).

In general, the technological process of wet seed dressing includes the following operations (Figure 1):

- Feeding of seeds into the mixing chamber.

- $\quad$ Preparation and feeding of the dressing product.

- $\quad$ Seed dressing (seed treatment with a disinfectant in a mixing chamber).

Removal of excess moisture and discharge of treated seeds into the vehicle.

Figure 2 uses the following designations:

- Clamp.

- Storage hopper.

- Seed dispenser.

- Liquid dispenser.

- Product dispenser. 
- Dressing agent preparation chamber.

- Dressing agent metering pump.

- $\quad$ Seed dressing chamber.

- Cyclone.

- Vehicle.

- Scraper conveyor.

- Screw conveyor.

- Grain chute.

- Dosing screw.

- $\quad$ Plate dispenser.

- Gate valve.

- Roller dispenser.

- Rotary dispenser.

- Centrifugal pump.

- $\quad$ Plunger-type pump.

- Gear-type pump.

- Centrifugal disk.

- Air flow.

- $\quad$ Auger.

- Screw conveyor.

- Scraper conveyor.

- Elevator.

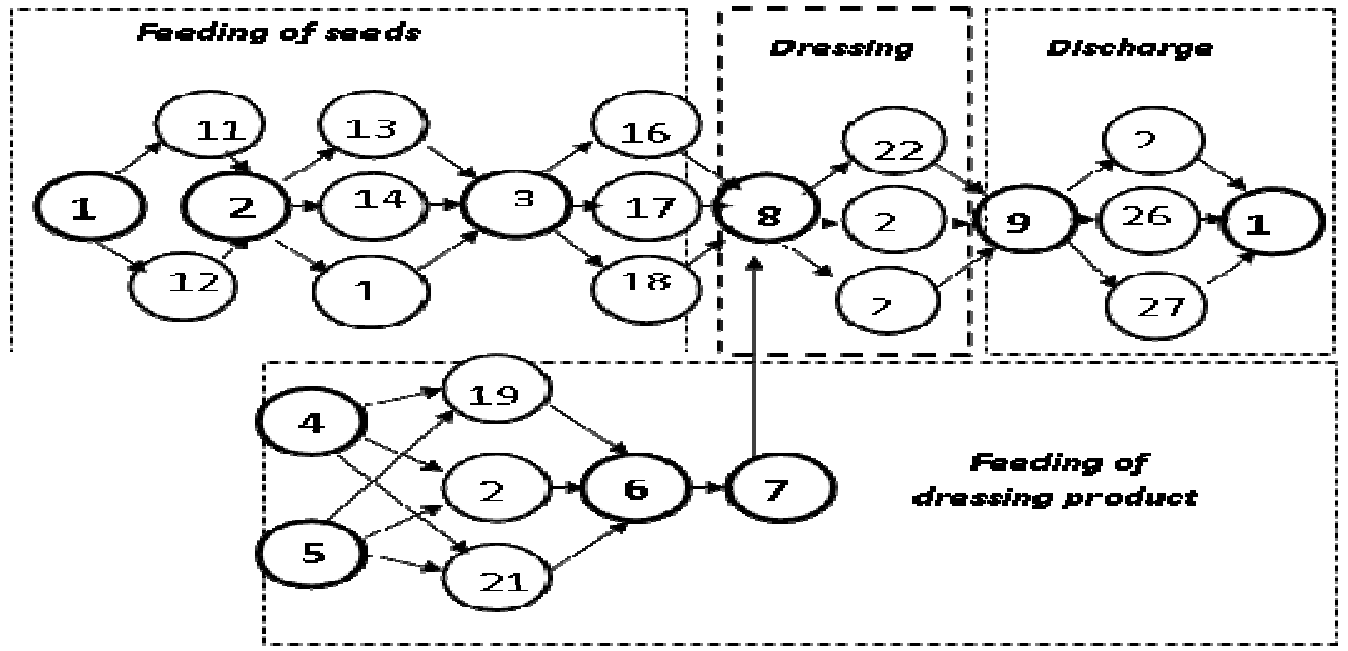

Figure 2: Technological Process Flow of Wet Seed Dressing.

The seed dressing process can be carried out by performing technological operations with the help of various working bodies. The analysis of the technological process of wet seed dressing shows that seeds can be transported from the clamp to the vehicle by various working bodies. The transportation of seeds from the clamp to the vehicle can be 
carried out according to various schemes.

scheme 1: 1-11-2-13-3-16-8-22-9-25-10

scheme 2: 1-12-2-14-3-17-8-23-9-26-10

scheme 3: 1-12-2-15-3-18-8-24-9-27-10.

The transportation of seeds from the clamp to the storage hopper can be carried out according to the scheme 111-2 (scraper conveyor) or 1-12-2 (screw conveyor).

According to the research results (Rosario, 1998; Wu, 1994), the damage of seeds of cereal and legume crops with standard moisture by scraper conveyors ranges from $0.4 \%$ to $0.6 \%$ and from 1.6 to $2 \%$, respectively.

Seed damage by the screw conveyors depends on the screw rotation speed, grain moisture, and conveyor performance. For cereal seeds, the damage to the kernels ranges from $2.3 \%$ to $17.3 \%$ (Liang, Wong, \& Nathan, 2000). For legumes (peas), it ranges from $15 \%$ to $25 \%$ (Kundu, 2002).

The seeds are fed from the hopper by a grain chute (13), auger (14), or a rotary apparatus (15) according to schemes $2-13-3 ; 2-14-3$ or $2-15-3$, respectively. The screw conveyor and the rotary apparatus, in addition to the functions of transportation, can simultaneously perform the functions of dosing. During transportation on a grain chute (scheme 2-13-3), the damage to the kernels usually does not exceed 1\% (Kundu, 2002).

When seeds are fed from the storage hopper to the dispenser according to the scheme 2-14-3, damage to the kernels occurs mainly in the loading zone and amounts to 3-5\% for cereal crops (Kundu \& Das, 2004), and up to 7\% for peas (Liang, Wong, \& Nathan, 2000).

Damage to the seeds by rotary machines (scheme 2-15-3) has been little studied, but we can assume that the principle of operation of these devices is similar to the operation of a roller machine. The crushing of seeds in roller machines ranges from 0.5 to $3.8 \%$ (Naphon, 2006).

The dosing of seeds entering to chamber 8 can be carried out according to the scheme $3-16-8$ with the help of a gate dispenser, according to the scheme 3-17-8 (roller dispenser) or according to the scheme 3-18-8 (rotary dispenser).

Gate dispensers belong to the devices for volumetric dispensing. The volume of material entering the chamber 8 depends on the size and shape of the metering hole, the speed of movement of the material, the type of the transporting working body. The considered schemes place of the technological process place such dispensers at the outlet of the grain chute (open or closed). Damage to the seeds does not exceed $0.5 \%$ due to the short length of transportation (Sharqawy \& Zubair, 2007).

Seeds are dressed in chamber 8 . Seeds and the dressing product are discharged into the seed dressing chamber according to one of the schemes (4-5-19-6-7), (4-5-20-6-7) or (4-5-21-6-7) depending on the design and principle of operation of the pumps.

The damage to the seeds during their coating with the dressing product is significantly influenced by the principle of operation, the type of working body distributing the seeds throughout the chamber, the physicomechanical properties of the material of the working body, the speed of interaction of the seeds with the walls of chamber 8 , friction, and aerodynamic and other physicomechanical properties of the treated culture. We shall consider the influence of the main 
types of valves (centrifugal disk, air flow, auger) on the damage to the seeds.

The centrifugal disk can be flat, conical, or spherical. To distribute the seeds in the chamber 8 , blades are used either mounted radially or at an angle to the radial position. Depending on the organization of the place of supply of seeds to a disk, the process of interaction of particles with a blade is accompanied by a blow. The speed of the blow pulse depends on the shape of the blades, the kinematic and geometrical parameters of the disk. The process of shock interaction of particles will be discussed below in more details. According to Sharqawy and Zubair (2008), micro and macro damage to the seeds by a centrifugal apparatus ranges from 1.5 to $2.8 \%$.

Damage to the seeds due to the shock interaction of particles with the steel walls of chamber 8 does not exceed 2\% (Kundu \& Barman, 2010). Thus, the total damage to the seeds in chamber 8 equipped with a seed dispenser in the form of a centrifugal disk is from 3.5 to $4.8 \%$.

The distribution of seeds in the chamber 8 by means of an air stream requires the installation on the machine of an additional working body-an air pump. Uniform distribution of seeds in the chamber requires higher air flow rate than the floating speed of the seeds during dressing. That is, the speed of the air flow must be controlled or exceed the floating speed of the densest seeds. To ensure the process of uniform distribution of seeds in the chamber 8 , it is recommended (Ziad, 2014) that the airflow should be at least $20 \mathrm{~m} / \mathrm{s}$. Ziad (2014) found that at $20 \mathrm{~m} / \mathrm{s}$ impact with the steel surface, the crushing of seeds was 3.8 to $4.2 \%$.

The use of a screw as a mixing device (SPSm-10 universal seed dressing machine and others) causes damage to seeds of cereal crops equal to 2.3-4.8\% (Kundu, 2002; Liang, Wong, \& Nathan, 2000). Damage to the seeds of legumes (peas) ranges from $5 \%$ to $10 \%$.

In practice, the discharge of seeds from a cyclone into a vehicle is carried out according to one of the schemes 925-10 (using a screw conveyor), 9-26-10 (scraper conveyor) or 9-26-10 (elevator).

As noted earlier (Rosario, 1998; Wu, 1994), damage to the seeds by a screw apparatus ranges from 2.3 to $17.3 \%$ and depends on the length of the transportation section, loading conditions, kinematic and geometrical parameters, and the type of crop. Damage to the seeds by the scraper conveyor is $0.4-0.6 \%$ (Rosario, 1998; Wu, 1994). The crushing of seeds with a bucket elevator is $0.7-3 \%$.

Given that, the above damage indicators are relative, since different authors accept different indicators, therefore, to assess the effect of the technological scheme on seed damage. Therefore, we take the average damage values for each type of working body. The degree of damage to seeds during their dressing is estimated by the sum of the ranks for each of the three schemes (Table 1).

Table 1: Damage to Cereal Seeds by Continuous Machines

\begin{tabular}{|c|c|c|c|c|}
\hline \multirow{2}{*}{ Process Operation } & \multirow{2}{*}{ Type of Working Body } & \multicolumn{4}{|c|}{ Damage to Seeds, \% } \\
\cline { 3 - 5 } & Schew conveyor & $2.3-17.3$ & - & Scheme 2 \\
\hline $1-11-2$ & Scheme 3 \\
\hline $1-2-2$ & Scraper conveyor & - & $0.4-0.6$ & - \\
\hline $2-13-3$ & Grain chute & $0.5-1.0$ & - & - \\
\hline $2-14-3$ & Dosing screw & - & $0.7 . .2 .5$ & - \\
\hline $2-15-3$ & Plate dispenser & - & - & 0.5 .2 .0 \\
\hline $3-16-8$ & Damper & $0.3-0.8$ & - & - \\
\hline $3-17-8$ & Roller dispenser & - & $0.3 . .0 .8$ & - \\
\hline $3-18-8$ & Rotary dispenser & - & - & $0.5-2.0$ \\
\hline
\end{tabular}




\begin{tabular}{|c|c|c|c|c|}
\hline \multicolumn{7}{|c|}{ Table 1: Contd., } \\
\hline $8-22-9$ & Centrifugal disk & $3.5-4.8$ & - & - \\
\hline $8-23-9$ & Air flow & - & $1.2-2.0$ & - \\
\hline $8-24-9$ & Distributing auger & - & - & $2.3-4.8$ \\
\hline $9-25-10$ & Screw conveyor & $2.3-17.3$ & - & - \\
\hline $9-26-10$ & Scraper conveyor & - & $0.4-0.6$ & - \\
\hline $9-27-10$ & Elevator & - & - & $0.7-1.5$ \\
\hline \multicolumn{7}{|c|}{} & $\mathbf{8 . 9}-\mathbf{4 1 . 2}$ & $\mathbf{4 . 0}-\mathbf{6 . 5}$ & $\mathbf{4 . 4}-\mathbf{1 0 . 9}$ \\
\hline
\end{tabular}

Table 1 shows that the damage to the cereal seeds during their dressing by continuous machines according to technological schemes similar to the PS-10A machine scheme is quite significant and ranges from 4.0 to $41.2 \%$.

Such damage to the seeds of cereals is due to the fact that different authors apply different indicators of damage. Some works present data on crushing, while other works consider macro and micro damages. In this case, maximum damage values are observed in technological schemes where screw conveyors are used. If the screw conveyors are replaced with other similar devices (scraper conveyor or elevator), then the total damage to the seeds can be reduced to $6.5-10.9 \%$. However, such damage to the seeds exceeds the permissible agro technical standards. Damage to the seeds of legumes by the working bodies of continuous machines is given in Table 2.

Table 2: Damage to Legume Seeds (Peas) by Continuous Machines

\begin{tabular}{|c|c|c|c|c|}
\hline \multirow{2}{*}{ Process Operation } & \multirow{2}{*}{ Type of Working Body } & \multicolumn{3}{|c|}{ Damage to Seeds, \% } \\
\cline { 3 - 5 } & Screw conveyor & $15-25$ & - & Scheme 1 \\
\hline $1-11-2$ & Scheme 2 & Scheme 3 \\
\hline $1-12-2$ & Grain chute & $1.0-2.5$ & - & - \\
\hline $2-13-3$ & Dosing screw & - & $2.0-3.5$ & - \\
\hline $2-14-3$ & Plate dispenser & - & - & 1.03 .0 \\
\hline $2-15-3$ & Damper & $2.0-5.0$ & - & - \\
\hline $3-16-8$ & Roller dispenser & - & $0.6-2.0$ & - \\
\hline $3-17-8$ & Rotary dispenser & - & - & $1.0-3.0$ \\
\hline $3-18-8$ & Centrifugal disk & $4.0-7.0$ & - & - \\
\hline $8-22-9$ & Air flow & - & $3.0-4.6$ & - \\
\hline $8-23-9$ & Distributing auger & - & - & $4.0-6.5$ \\
\hline $8-24-9$ & Screw conveyor & $15-25$ & - & - \\
\hline $9-25-10$ & Scraper conveyor & - & $1.6-2.0$ & - \\
\hline $9-26-10$ & Elevator & - & - & $1.0-3.0$ \\
\hline $9-27-10$ & & $\mathbf{3 7 . 0 - 6 4 . 5}$ & $\mathbf{8 . 8}-\mathbf{1 3 . 1}$ & $\mathbf{8 . 6}-\mathbf{1 7 . 5}$ \\
\hline
\end{tabular}

Analysis of data in Table 2 shows that the damages of pea seeds by the working bodies of continuous machines exceed those of cereals by $1.5-3.0$ times or more.

This indicates that the technological process of dressing requires improvement in the form of reducing the number of working bodies interacting with the seeds, as well as in the search of materials for the working surfaces of the machines that would minimize damage to seeds.

The first improvement is implemented in continuous machines (SPSm-10, PS-10, and others created on their basis) having a minimal number of working bodies.

Figure 3 shows the flowchart of the SPSm-10 machine for seed dressing. The technological process of this machine is close to that as shown in Figure 1 but differs in a simpler design and method of mixing seeds with a dressing product. 
The material is withdrawn from the clamp 1 by the screw 2 and transported to the storage hopper by the scraper conveyor 3. The seeds are fed into the mixing chamber 9 by a damper 4 . The dressing product is fed to the chamber 9 according to the previously described scheme, dosing of water 5 , dosing of the dressing product 6 , preparation of the solution 7 (mixing) and feeding 8 of the spray material into the mixing chamber 9.

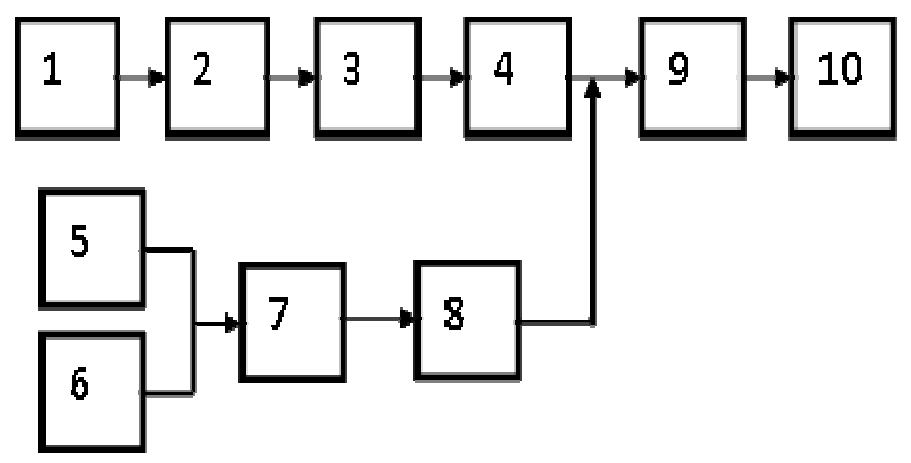

Figure 3: Technological Process Flow of Seed Dressing by SPSm-10 Machines.

Since the technological process of these machines involves no device for removing excess moisture, the working bodies transporting the processed material from the mixing chamber to the cyclone are excluded. This allows in reducing slightly the degree of damage to the seeds.

The transportation of seeds from the clamp to the vehicle is carried out in accordance with the scheme 1-2-3-49-10. At the same time, the distribution of seeds through the mixing chamber 9 is performed by a centrifugal disk, and the process of transporting the treated seeds $9-10$ is accompanied by additional mixing. Damage to the seeds of cereals and legumes by the working bodies of these machines is given in Table 3.

Table 3: Damage to the Seeds of Cereals and Legumes by an SPSm-10 Machine

\begin{tabular}{|c|c|c|c|}
\hline \multirow{2}{*}{ Process operation } & \multirow{2}{*}{ Type of working body } & \multicolumn{2}{|c|}{ Damage to seeds, \% } \\
\cline { 3 - 4 } & & cereals & Legumes \\
\hline $1-2$ & Screw conveyor & $2.3-17.3$ & $15-25$ \\
\hline $2-3$ & Scraper conveyor & $0.4-0.6$ & $1.6-2.0$ \\
\hline $3-4$ & Grain chute & $0.5-1.0$ & $1.5-2.5$ \\
\hline $4-9$ & Damper & $0.3-0.8$ & $2.0-5.0$ \\
\hline 9 & Centrifugal disk & $3.5-4.8$ & $4.0-7.0$ \\
\hline $9-10$ & Screw conveyor & $2.3-17.3$ & $15-25$ \\
\hline & $\mathbf{\Delta} \boldsymbol{\Pi}$ & $\mathbf{9 . 3}-\mathbf{4 1 . 8}$ & $\mathbf{3 9 . 1}-\mathbf{6 6 . 5}$ \\
\hline
\end{tabular}

During the implementation of the process (Figure 3) 1-2-3-4-9-10, damage to the seeds of cereals is from 9.3 to $41.8 \%$, and damage to the seeds of peas can reach $\mathbf{\Sigma} \Delta \mathbf{P}=66.5 \%$.

\section{CONCLUSIONS}

The analysis of the pre-sowing technological process with continuous machines showed that screw working bodies have the greatest impact on seed damage during pre-sowing treatment. Damage to the seeds by screw working bodies ranges from 34.6 to $50.0 \%$ of the treated seeds.

\section{SUMMARY}

To reduce the loss of seeds of cereals and legumes, it is necessary to exclude the use of screw working bodies in the process of pre-sowing treatment of seeds. 
The second way to reduce grain losses due to their damage can be implemented through the use of materials with high elastic properties (elastomers) or the like.

The use of elastomers for the manufacture of working bodies requires additional research aimed at substantiating the scope of their application, the requirements to ensure their durability, design, and process parameters.

\section{ACKNOWLEDGEMENT}

This research was carried out with the financial support of the RFBR grant, project No. 19-01-00250 and No. 17-0100323 .

\section{REFERENCES}

1. L. Rosario, M. M. Rahman, "Overall Efficiency of a Radial Fin Assembly under Dehumidifying Conditions", ASME, Journal of Energy Resources Technology120, (1998) 299-304.

2. G. Wu, T. Y. Bong, "Overall Efficiency of a Straight Fin with Combined Heat and Mass Transfer", ASHRAE Transactions 100 (1994) 367-374, part I.

3. S. Y. Liang, T. N. Wong, G. K. Nathan, "Comparison of One-Dimensional and Two-Dimensional Models for Wet-Surface Fin Efficiency of a Plate-Fin-Tube Heat Exchanger", Appl. Thermal Eng. 20 (2000) 941-962.

4. B. Kundu, "An Analytical Study of the Effect of Dehumidification of Air on the Performance and Optimization of Straight Tapered Fins", Int. Commun. Heat Mass Transfer 29 (2002) 269-278.

5. B. Kundu, P. K. Das, "Performance and Optimization Analysis for Fins of Straight Taper with Simultaneous Heat and Mass Transfer", Transaction of the ASME 126 (2004) 862-868.

6. P. Naphon, "Study on the Heat Transfer Characteristics of the Annular Fin under Dry-Surface, Partially Wet-Surface, and Fully Wet-Surface Conditions", International Communications in Heat and Mass Transfer 33 (2006) 112-121.

7. M. H. Sharqawy, S. M. Zubair, "Efficiency and Optimization of Longitudinal Fin with Combined Heat and Mass Transfer-an analytical solution", Int. J. Refrig. 30 (2007), 751-757.

8. M. H. Sharqawy, S. M. Zubair, "Combined Heat and Mass Transfer Analysis from Annular Fins of Constant Cross-Sectional Area", in: 8th International Symposium on Advances in Computational Heat Transfer Proceedings, ICHMT, Marrakech, Morocco, Paper\# CHT-08-239, May 2008.

9. B. Kundu and D. Barman, "Analytical Study on Design Analysis of Annular Fins under Dehumidifying Conditions with a Polynomial Relationship Between Humidity Ratio and Saturation Temperature", International Journal Heat and Fluid Flow, 2010 .

10. Ziad, M. Almakhyoul," Heat Characteristics and Performance of Longitudinal Fin Exposed to Wet Air", Al-Rafidain Engineering Journal, Vol.22, No.3, 2014. 